\title{
X-ray applications and recent advances @ XLab Frascati
}

\author{
S. B. Dabagov ${ }^{1,2,3}$ - D. Hampai ${ }^{1}$ - V. Guglielmotti ${ }^{1} \cdot$ G. Cappuccio $^{1} \cdot$ E. Capitolo $^{1} \cdot$ Yu Gladkikh $^{4}$
}

Received: 5 November 2019 / Accepted: 17 April 2020 / Published online: 13 May 2020

(c) Accademia Nazionale dei Lincei 2020

\begin{abstract}
$\mathrm{XLab}$ Frascati is a facility open to external users for different X-ray analyses, ranging from structural studies through X-ray diffraction to elemental mapping by means of $\mu \mathrm{X}$-ray Fluorescence, colour tomography and X-ray imaging studies. This is possible thanks to our experimental layouts XENA (X-ray Experimental station for Non-destructive Analysis) and RXR (Rainbow X-Ray) along with our facility for the production of polycapillary optics. The know-how on these optics enable us improving the performances of our setups by adopting the best fitting X-ray optics according to the experimental requirements. To make an example, the main advantage of RXR is that the detection system includes two spectrometers working in high (arranged in the polycapillary confocal geometry) and low X-ray energies, respectively, permitting both the 2D $\mu \mathrm{XRF}$ scan and 3D $\mu \mathrm{XRF}$ elemental mapping (colour tomography) due to the confocal geometry with a 3-axis fine motion system. The article showcases the results obtained in key case studies where we performed: (i) $2 \mathrm{D} / 3 \mathrm{D} \mu \mathrm{XRF}$ to analyse chemical composition of tree rings with respect the influence of the environmental context, to study a "fresco" fragment and assess the presence of damages, to make a 3D reconstruction of a screw encapsulated in glue through the its elemental composition as well as (ii) X-ray imaging and (iii) $\mu \mathrm{CT} /$ dynamic $\mathrm{CT}$ to characterize $\mathrm{LiF}$ detectors, to study the structure of a flower bud and to evaluate the profile distribution of a diesel spray from the nozzle.
\end{abstract}

Keywords X-ray imaging $\cdot$ microXRF $\cdot$ Polycapillary optics $\cdot$ Mapping

\section{Introduction}

For the past 15 years, at the Laboratori Nazionali di Frascati (LNF-INFN) a team of researchers has been focused on the study of X-ray optics, in particular polycapillary optical elements, being involved in several national and international projects and collaborations. The result of this research activity resulted in the setting up of the XLab Frascati (XlabF) laboratory, officially inaugurated in the year 2010, and that

This contribution is a peer-reviewed version of a paper presented at the international meeting of the non destructive techniques on cultural heritage (NDT-CH 2018) held October 12, 2018 in Buenos Aires ( Argentina).

S. B. Dabagov

sultan.dabagov@lnf.infn.it

INFN-LNF, XLab Frascati, Frascati, Rome, Italy

2 National Research Nuclear University MEPhI, Moscow, Russia

3 RAS P.N. Lebedev Physical Institute, Moscow, Russia

4 Belgorod State University, Belgorod, Russia was aimed at the characterization of novel optics (in particular polycapillary optics) and the building up of different experimental schemes for various $\mathrm{X}$-ray applications such as X-ray diffraction (XRD), X-ray fluorescence (XRF) and total reflection X-ray fluorescence (TXRF) and X-ray imaging. The term " $\mathrm{X}$ " in the $\mathrm{XlabF}$ abbreviation does not just indicate the name of the radiation, but it also represents a crossroad of interactions: XLab Frascati is involved in a number of projects (UA9-LUA9, GEMINI, POSSO, MicroX, POLYX, NANORAY, GMINUS2) within collaboration programs (Universities, Research Institutes and industrial partners) focused on shaping up charged and neutral particle beams by means of various techniques such as crystal channeling, channeling in laser and plasma fields, capillary/polycapillary optics. ${ }^{1}$ Thanks to the experimental setups available at XLab Frascati that are currently open to the users, we offer the possibility to perform both elemental and structural studies based on the application of the

\footnotetext{
${ }^{1}$ PolyCapillary optics (also named PolyCO) are glass-based optical devices (lenses and/or semilenses) composed of millions of micro (or submicron) channels, in which the trapped radiation is efficiently transmitted by multiple reflections, thus allowing the user to obtain a focused or parallel brilliant X-ray source.
} 
Table 1 The features summary for the XENA and RXR facilities

\begin{tabular}{|c|c|c|}
\hline & Type of analysis & Technique resolution \\
\hline \multirow[t]{5}{*}{ XENA } & High-resolution imaging & $<1 \mu \mathrm{m}$ (with LiF detector) \\
\hline & $\mu \mathrm{CT}$ & $\begin{array}{l}<17 \times 17 \times 17 \mu \mathrm{m}^{3}(\mathrm{CT} \text { with spatial resolution } \mathrm{CCD} \text { cam- } \\
\left.\text { era of } 10.4 \times 10.4 \mu \mathrm{m}^{2}\right)\end{array}$ \\
\hline & $\mathrm{X}$-ray optics characterization & \\
\hline & Detector characterization & \\
\hline & $\begin{array}{l}\text { Novel sources (i.e. cold cathodes } \\
\text { for X-ray tubes) }\end{array}$ & \\
\hline \multirow[t]{3}{*}{ RXR } & $\mu \mathrm{XRF} 2 \mathrm{D}$ mapping & $<80 \times 80 \mu \mathrm{m}^{2}$ \\
\hline & $\mu \mathrm{XRF} 3 \mathrm{D}$ mapping & $<80 \times 80 \times 80 \mu \mathrm{m}^{3}$ (step resolution combined with polyCO) \\
\hline & TXRF & $25 \pm 1.25 \mathrm{ng} / \mathrm{g}$ concentration \\
\hline
\end{tabular}

aforementioned X-ray optics in several fields such as cultural heritage, innovative materials, medical diagnostics, pharmacology, beam diagnostics, detectors characterization, to name a few. In addition, XlabF has the unique capability to realize polycapillary optics, thanks to the presence of the Polycapillary Technopolo "XChannel" located within the INFN National Laboratories of Frascati and completely dedicated to the complete manufacturing of lenses and semilenses from raw materials and their characterization in terms of X-ray transmission and gain. In this respect, the established expertise and the know-how of the XlabF researchers on polycapillary optics enables us to design and produce customised optics as well as the development of new instrumental prototypes and new X-ray desktop facilities for carrying out advanced studies following the user requirements. The paper will show the main results obtained with classical X-ray techniques (XRD, XRF, X-ray Imaging and X-ray computed tomography) composed by polycapillary optical elements and, in particular, the advances for the analysis of low-contrast samples like biological specimens and injection sprays from engine's injection devices.

\section{Research activities and facilities available @ XLab Frascati}

The main research activities performed at XlabF are focused on X-ray analysis by means of desktop techniques, mostly based on polycapillary optical elements, and on theoretical studies of charged and neutral particles interactions in different fields, especially concerning channelling, the field of physics that studies the path of charged particles or electromagnetic radiation inside regular structures such as crystals, for instance. In particular, our laboratory equipment is dedicated to several applications ranging from the analysis of micro-macro $\mathrm{X}$ fluorescence (traditional, confocal, total external reflection) applied on Cultural Heritage and Geological samples to X-ray diffraction applied to the examination of materials as well as temporary experiments and testing systems for the design of new detectors and study of imaging techniques along with $\mathrm{X}$ tomography projects. Presently, there are two facility stations - XENA and RXR - that are open to users and allow the combination of different elemental and imaging techniques with the purpose of obtaining a complete X-ray analysis of the sample under investigation. XENA (X-ray Experimental station for Non-destructive Analysis), that is operative since 2004, is a facility dedicated exclusively to imaging, tomography and characterization of X-ray devices such as novel sources such as cold cathodes for X-ray generation based on carbon nanotubes and nanostructures (NANORAY 2011), optics (Hampai et al. 2009)_diffractive crystals (Gogolev et al. 2015a) and vibrating systems (Liedl et al. 2015) - as well as detectors (Gogolev et al. 2015b). Our layout is based on the combination of three X-ray Oxford Apogee tubes (W, Mo and $\mathrm{Cu}$ anodes), a set of mechanical components and motors for lens alignment and scanning, and an optical table providing many geometrical setup possibilities. RXR (Rainbow $\mathrm{X}$-Ray) is an optimized system of previous versions (Hampai et al. 2008, 2014, 2018) dedicated to performing 2D/3D XRF micro-imaging and TXRF (Total Reflection X-ray Fluorescence) analysis. It is equipped with two detectors of different energy efficiency aiming at measuring a full spectrum ranging from $800 \mathrm{eV}$ to $25 \mathrm{keV}$. RXR typically works in confocal mode where the source is coupled with a full-lens while both the detectors are combined with dedicated half-lenses. Furthermore, RXR is equipped with an (optional) vacuum chamber enabling the user to carry out measurements in the low-energy range, as required in specific cases, and to insert a polyCO (polyCapillary optics) when TXRF analysis is performed. In Table 1, we give an overview of the key characteristics of the XENA and RXR facilities that are currently running at XLab Frascati. 


\section{X-ray fluorescence (XRF) case studies}

To show the RXR potentialities, we describe three application scenarios where we performed XRF studies to determine the presence of specific chemical elements with respect to their concentration and position within the samples and to make 3D reconstruction of a solid object: (a) analysis of tree rings to evaluate the influence of environmental context, (b) the study of a "fresco" fragment to assess the presence of damages and (c) the 3D- $\mu$ XRF reconstruction of a screw encapsulated in glue based on the elemental composition.

\subsection{Tree rings analysis}

Several natural archives may provide unaltered records of environmental and climatic information at different time scales dating back up to several hundred thousand years: marine sediments (Bostick et al. 2005; Moy et al. 2011), stalagmite conformations (Polyak and Asmerom 2001), tree rings (Bernini et al. 2016) and deep ice cores (Petit et al. 1999; Community Members EPICA 2004; Maggi 1997) to name a few.

For instance, the analysis of tree rings is a powerful historical ecology tool due to the inter- and intra-annual responses of the radial growth to local climatic variations. In particular, the study of correlation and response functions among tree rings with monthly/weekly climatic factors have been successfully assessed during the past years. Moreover, dendrochemical analyses have shown the possibility of detecting the accumulation of contaminants and pollutants in wood samples during the time (Bernini et al. 2016; Filippo et al. 2007).

In this respect, measuring the chemical component concentrations in tree rings has powerful implications both for biological and ecological studies. In fact, information on the spatial/temporal distribution within woody tissues of chemical elements (such as $\mathrm{Ti}, \mathrm{Ca}$ and $\mathrm{K}$ ) that are considered both key components of wood cells as well as nutrients involved in basic physiological cellular processes, can provide deep insights into how trees allocate nutrients in their tissues according to species, age, or other different environmental contexts.

In addition, the capability of trees to store in their annual rings both inorganic (e.g. lead) and organic (e.g. $\mathrm{HCH}$ ) pollutants can provide an ex post monitoring tool to quantitatively reconstruct the time dynamics of pollution over a territory (Bernini et al. 2016; Sawidis et al. 2011).

We mention here the results we obtained during the 2D $\mu \mathrm{XRF}$ mapping of a set of samples from different species belonging to both conifers and angiosperms. In particular,

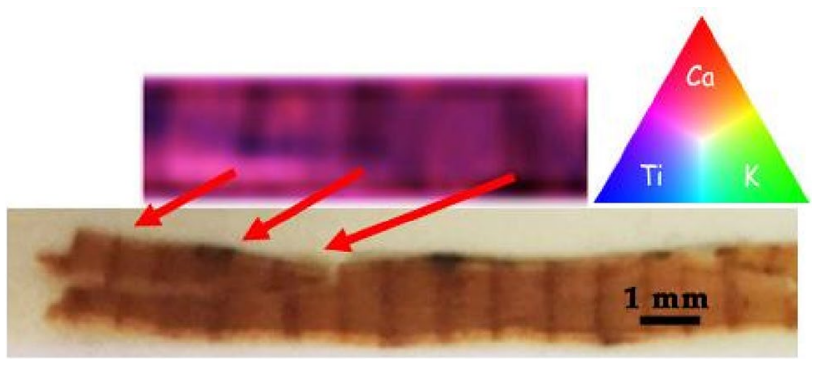

Fig. 1 Section of the 2D mapping $\mu$ XRF. Both $\mathrm{Ca}$ (red) and Ti (blue) reproduce, in terms of their spatial accumulation, the time sequence of tree rings as visible in the optical image indicated by the red arrows (Cappuccio et al. 2018)

we set up a preparation procedure for analysis for the entire core and for a slice with a thickness of around $500 \mu \mathrm{m}$ that was based on making acquisition by using $100 \times 100 \mu \mathrm{m}^{2}$ steps with $10 \mathrm{~s} / \mathrm{step}$ acquisition time.

In Fig. 1 we depict, by a three-colour scale, the localization of high $\mathrm{Z}$ elements such as $\mathrm{Ca}$, Ti and of $\mathrm{K}$, as they are all basic elements normally present in wood. In particular, the red and blue areas representing $\mathrm{Ca}$ and $\mathrm{Ti}$, respectively, reproduced the sequence of different bands of earlywood and latewood due to their perfect match with their occurrence in tree rings when compared to the optical image of the studied sample. These findings are a confirmation of the location of these chemical elements, as inclusions present in the tree rings analysed, on a temporal scale as rings are normally used to date trees.

\subsection{Fresco fragment study}

We performed a set of tests on an archaeological sample, i.e. a "fresco" fragment, coming from a villa of the late nineteenth century (Fig. 2a). To carry out these measurements, we analysed a surface of $20 \times 20 \mathrm{~mm}^{2}$ with measurement step $\Delta x=\Delta y=250 \mu \mathrm{m}$ (Fig. 2b), an acquisition time of $5 \mathrm{~s} /$ step, using an X-ray source of Mo $(35 \mathrm{kV}$ and $800 \mu \mathrm{A})$.

In Fig. 2c we show the fluorescence analysis we recorded, where the intensity of each pixel is obtained through the integration over a limited range of the spectrum from 1 to $15 \mathrm{keV}$.

The intensity values are represented by a rainbow colour scale and, although we used big steps, it is clearly evident that the pattern in the image of Fig. $2 b$ (obtained at visible frequencies) is completely reproduced by the XRF map.

Moreover, the Fig. 2d and e depict the $\mu$ XRF map, through the RGB scale, obtained by collecting the intensity related to three selected elements such the triplet $\mathrm{Ti} / \mathrm{Fe} / \mathrm{Co}$ and $\mathrm{Mn} / \mathrm{Zn} / \mathrm{Cu}$, respectively.

In particular, Fig. 2d highlighted the strong presence of $\mathrm{Ti}$ and Fe while the Co is likely to be scarcely present and 

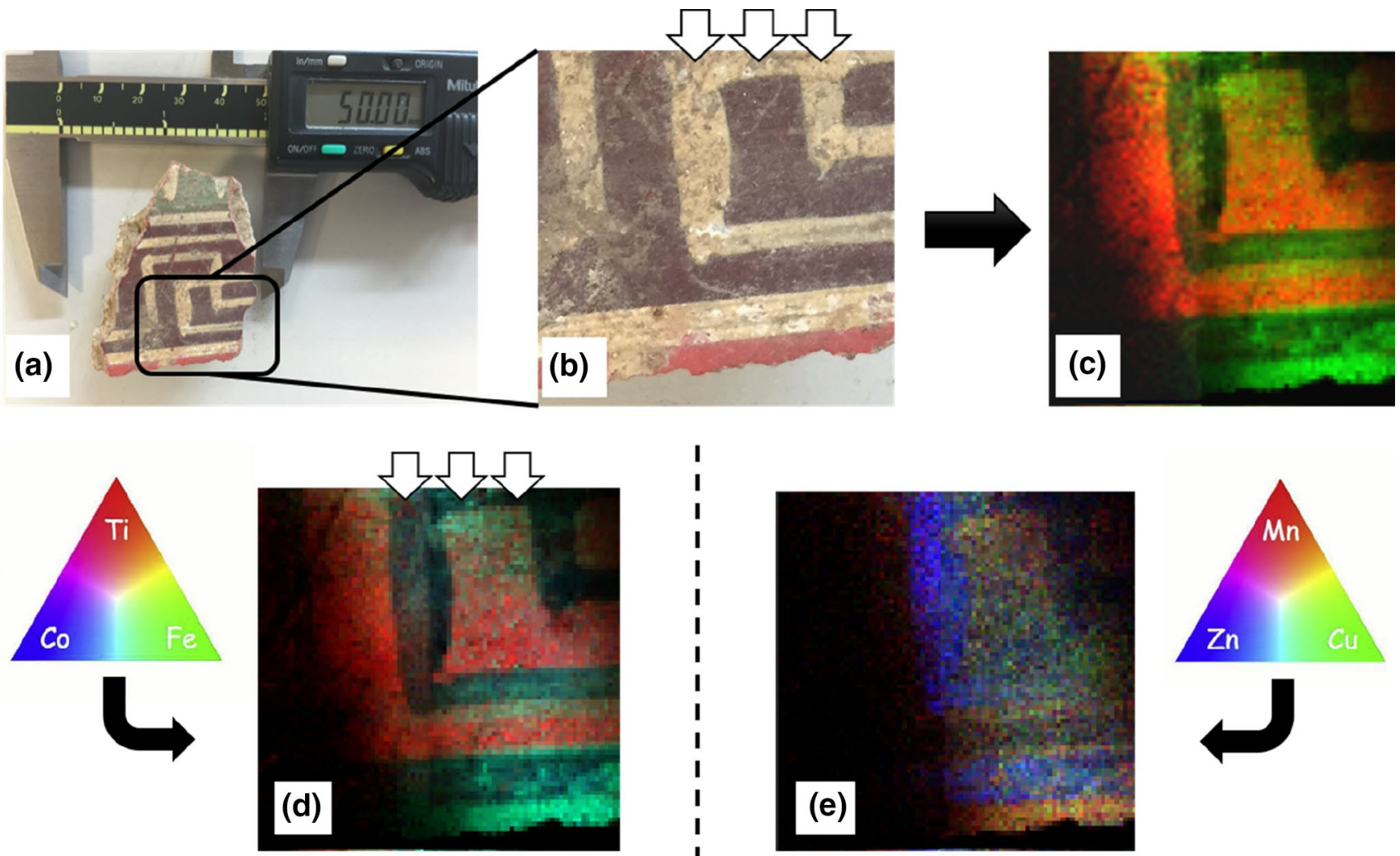

Fig. 2: $2 \mathrm{D}-\mu \mathrm{XRF}$ mapping analysis of fresco fragment from a late nineteen Villa: a Image of the sample; b The scanned area; c $2 \mathrm{D}-\mu \mathrm{XRF}$ scanning maps: analysed area of $20 \times 20 \mathrm{~mm}^{2}$, step $250 \times 250 \mu \mathrm{m}^{2}$, acquisition time $5 \mathrm{~s} / \mathrm{step}, \mathrm{X}$-ray source Mo $(35 \mathrm{kV}$,

hence difficult to be visually recognised. At the same time, Fig. 2e allowed us to deduct that ochre colour of the Greekfret recognizable in the visible region, is mainly composed of $\mathrm{Zn}$, while the presence of $\mathrm{Mn}$ and $\mathrm{Cu}$ cannot be excluded.

Moreover, from the comparison of the optical image of the sample with the RGB image of the Fig. 2d, we evidenced the absence of $\mathrm{Ti}$ in the outermost layer located in the upper part of the mapped sample (as indicated by the white arrows) that lead to the hypotheses of damage caused during the extraction of the sample from the its original location.

We may also infer that, under construction, the red painting composed mainly by Iron, was applied onto the base before the application of the subsequent colours.

\subsection{D reconstruction and elemental recognition of a hard-metallic sample}

Conventional X-ray tomography techniques allow getting three-dimensional reconstructions, even of large objects, with sub-millimetre resolutions by keeping high contrast also for those objects composed by various inclusions characterized by very different density. However, the
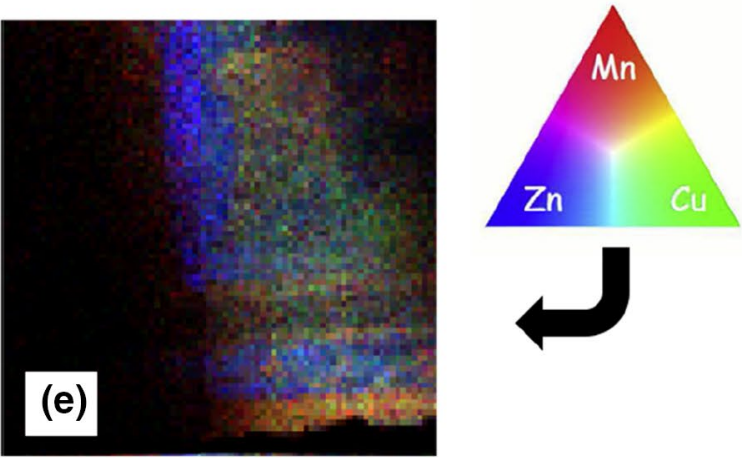

$800 \mu \mathrm{A}) ; \mathbf{d}$ and $\mathbf{e}$ By means of inverse colours the elements present in a sample are highlighted (the triplet $\mathrm{Ti} / \mathrm{Co} / \mathrm{Fe}$ and the triplet $\mathrm{Mn} / \mathrm{Zn} /$ $\mathrm{Cu}$ ) (Hampai et al., 2017). The white arrows indicate the region of $\mathrm{Ti}$ absence probably due to damage caused during the sample extraction

information obtained are mostly morphological without any elemental data easy to access. At the same time, since conventional micro-fluorescence instruments do not utilize a confocal scheme, no bulk information, morphological and elemental data are provided.

In this respect and thanks to the ellipsoidal spot of about $80 \div 90 \mu \mathrm{m}$, RXR enables the user in getting 3D bulk information. In order to show the confocal geometry capabilities as well as to discriminate volumes characterized by different densities and composition, we have investigated a sample including a hard-metallic piece surrounded by a soft solid medium: we have prepared a sample defined by a screw encapsulated in a drop of ethanol and methyl acetate glue.

Unlike a classical tomography approach (i.e. CT scan), $3 \mathrm{D}-\mu \mathrm{XRF}$ reconstruction has been performed collecting $2 \mathrm{D}$ scans on different sections along the axial direction. During our measurements a total volume of $4.1 \times 4.1 \times 2.1 \mathrm{~mm}^{3}$ was probed with a step of $100 \mu \mathrm{m}(\Delta x=\Delta y=\Delta z)$ and an acquisition period of $5 \mathrm{~s} / \mathrm{step}$.

As an example, in Fig. 3 the images of two different layers have been compared: the first was taken in the lower part of 

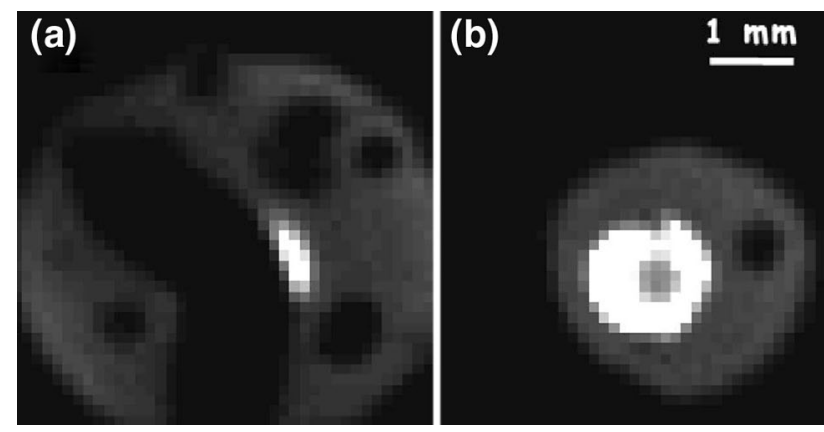

Fig. 3: $2 \mathrm{D}$ scan of a metal screw encapsulated in the glue. a The first layer refers to the lower volume of the $3 \mathrm{D}$ scan and $\mathbf{b}$ the second layer has been obtained by scanning slightly below the top surface of the screw. Images clearly show three different regions: a high fluorescence due to the presence of the metal screw (white area), a region associated with the primary radiation scattered by the glue (grey area) and a signal-free region (black area) (Hampai et al. 2018)

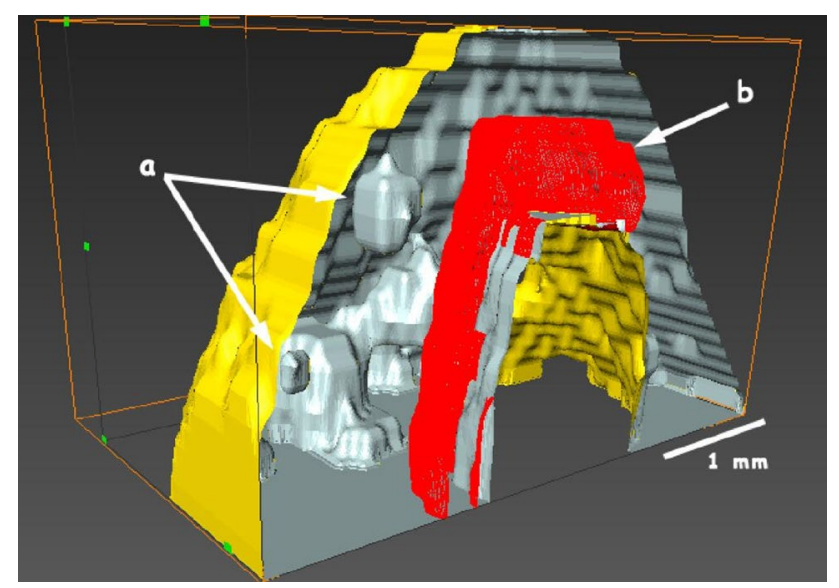

Fig. 4: 3D reconstruction section of a screw encapsulated in the glue: the arrows a identify two air bubbles trapped during the curing process of the glue while $\mathbf{b}$ identifies the location of the screw (Chang et al. 2001)

the sample volume while the second was collected in the region corresponding to the screw end.

As clearly visible, the scan performed was able to reveal three regions depicted through a grey-scale of the X-ray intensity: the first two are due to the fluorescence of the metal screw embedded in the glue (white area) and the radiation scattered by the soft matter (grey area), while the third is a set of regions with no signal registered and that were associated with the presence of air bubbles trapped during the curing process of the glue (black areas).

As anticipated 3D XRF technique allows the recognition of specific elemental contributions when compared to tomography and Fig. 4 perfectly demonstrates this advantage: we can recognize the iron contribution outlined in red, the external surface of the scattered beam in yellow and the internal surface of the sample in grey through the $3 \mathrm{D}$ rendering. In addition, this "elemental" visualization, with a spatial resolution of $100 \mu \mathrm{m}$, clearly highlights the presence of bubbles (indicated by the two arrows "a") formed during the curing process of the glue while arrow $b$ indicates the presence of the screw.

\section{X-ray based techniques and analysis of case studies}

The following paragraphs summarize the results obtained with the application of X-ray analysis on different case studies through the use of our XENA facility. In particular, we focused on three key techniques that were implemented and optimised thanks to the use of polycapillary optics such as $\mathrm{X}$-ray imaging, micro computed tomography $(\mu \mathrm{CT})$ and dynamic computed tomography (dynamic CT).

\subsection{X-ray imaging}

X-ray imaging techniques play a crucial role in understanding the microscopic world: the impressive improvements in all three key elements, X-ray sources, optics and detectors, which form the basis of this technology, make it a powerful tool in life science (Kirz and Jacobsen 1995) and biology (Cotton et al. 2015), as well as in materials science, industrial and medical applications.

With the advent of synchrotron sources, sophisticated $\mathrm{X}$ microscopy configurations based on projection methods have been exploited to overcome the poor spatial resolution of the standard X-ray imaging detectors, like CCD cameras. However, the development of laboratory table-top X-ray microscopy systems dedicated to X-ray imaging is highly desirable.

In this respect, novel LiF-based X-ray imaging detectors have been proposed and tested for soft X-rays (up to 1-1.5 keV) (Bonfigli et al. 2007; Baldacchini et al. 2003) and for hard X-rays (up to about $8 \mathrm{keV}$ ) (Almaviva et al. 2006) given their peculiar feature. In fact, they possess very high spatial resolution over a large field of view, wide dynamic range, versatility and simplicity of use and sensitivity of the optical fluorescence reading technique, thus making them very promising and attractive for $\mathrm{X}$ imaging applications and the development of easy-to-use (table-top) X-ray microscopy systems (Fukuda et al. 2008).

The intrinsic spatial resolution of the LiF-based imaging plate is related to the physical dimensions of point defects, better known as colour centres (CCs) that are comparable with atomic scale (about $1 \mathrm{~nm}$ ), although it is limited by the optical microscope characteristics and the fluorescence detection technique utilized in the reading system.

In this respect, the combination of polycapillary lens and fine-focused micro X-ray tube (with a source spot size less 
Fig. 5 a Optical image under white light illumination in transmission mode of a doubly polished fragment of a magnesiumhastingsite amphibole (sample 2); b and $\mathbf{c} X$-ray radiography stored on a LiF crystal and read by the CLSM in fluorescence mode; $\mathbf{d}$ Shows the intensity profile along a dark dot with a bright rim (line L) with corresponding FWHM of the dark dot prole (Hampai et al. 2011)

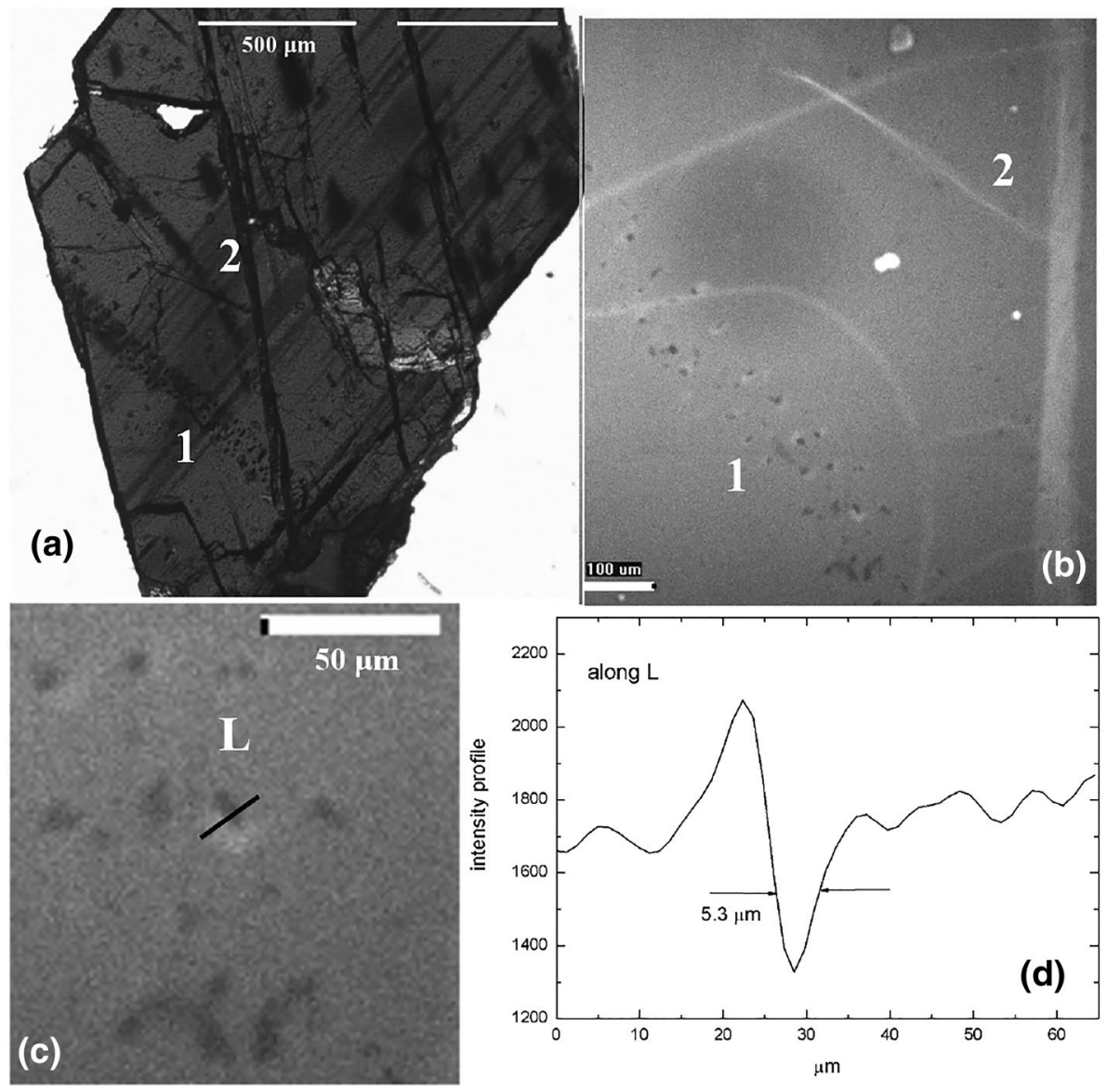

than 50 microns in diameter) can provide the high-intensity radiation flux adequate to perform a very high-resolution imaging of a sample. In comparison to a pinhole (Chang et al. 2001), an optimized "X-ray source-optics" system can result in radiation density gain (the ratio of X-ray intensities with and without the optics at the focal spot) of more than 3 orders of magnitude (Dabagov 2003; Hampai et al. 2007).

As an example of this powerful technique, we report the results obtained on a sample made of a doubly polished fragment of a magnesium-hastingsite amphibole, a complex rock-forming silicate with the ideal composition $\mathrm{NaCa}_{2}$ $\left(\mathrm{Mg}_{4} \mathrm{Fe}^{3+}\right)\left(\mathrm{Si}_{6} \mathrm{Al}_{2}\right) \mathrm{O}_{22}(\mathrm{OH}, \mathrm{O})_{2}$. This specimen typically occurs in nature as megacrystals within a gabbroid pluton from Fuegian Andes (Argentina) and displays abundant pseudo-secondary and primary fluid inclusions (Liedl et al. 2015). FTIR (Fourier Transform InfraRed spectroscopy), EMP (Electron MicroProbe) and textural analyses of the primary fluid inclusions indicate the presence of $\mathrm{CO}_{2}$ and tiny magnetite crystals (Ridolfi et al. 2010).

Figure 5 shows the details extracted from our analysis performed on the sample: Fig 5a shows the images of the sample, directly obtained by the optical microscope under white light illumination in transmission mode while Fig. 5b shows its X-ray micro-radiography stored in a LiF crystal and read by the CLSM (Confocal Laser Scanning Microscopy) in fluorescence mode.

Higher absorbing areas in the optical and X images (1) could be assigned to magnetite (that is mainly composed of $\mathrm{Fe}^{3+}{ }_{2} \mathrm{Fe}^{2+} \mathrm{O}_{4}$ ), which is known to be finely dispersed within the crystal, while transparent structures in the $\mathrm{X}$ image (2), absorbing in the optical image, could be due to fluid inclusions containing $\mathrm{CO}_{2}$ and/or $\mathrm{H}_{2} \mathrm{O}$ which have been identified by FTIR microspectroscopy (Ridolfi et al. 2010). Moreover, in the $X$ micro-radiography, several absorbing dots present brighter rims, as shown in the magnified image in Fig. 5c and Fig. 5d reports the intensity profile along a dark dot with a bright rim (line L) with the corresponding FWHM of the dark-dot profile.

The plot indeed evidences the different $\mathrm{X}$ absorption properties between the dark structures and their edges, a peculiar feature which is not resolved in the optical images. In the LiF-based X-ray micro-radiography (Fig. 5b) few mechanical scratches on LiF crystal surfaces are present, due to the polishing procedure. Good-optical-quality LiF film-based detectors (Montereali 2002) overcome this disadvantage and allow to collect better-quality and artefactfree fluorescence images (Montereali et al. 2010). 


\subsection{Micro X-ray computed tomography $(\mu \mathrm{CT})$}

In this experiment, we report the results obtained to reconstruct a flower bud through the X-ray micro-tomography technique.

The X-ray tomography test bench was composed of a radiation source, an Oxford Apogee 5000 tube $(\mathrm{Cu} \mathrm{Ka})$, with a source spot of about $50 \times 50 \mu \mathrm{m}^{2}$ and power of $50 \mathrm{~W}$, a Photonic Science CCD (FDI 1:1.61) with a pixel resolution of $10.4 \times 10.4 \mu \mathrm{m}^{2}$ and a Newport micrometre positioner xyz 9 .

To obtain a rather small radiation divergence, which allows getting a very small blurring effect, we have used a polycapillary semilens.

As known, any lens is characterized by a residual divergence responsible for the resolution of the image to be reconstructed by microCT and to estimate that, we consider that the sample dimension $\Delta \mathrm{X}_{0}$ is reproduced at the detector as $\Delta \mathrm{X}$, so as the effect can be estimated as $\left|\Delta X-\Delta X_{0}\right| / 1 \Delta \theta$, where 1 is the distance from the sample to the detector. As a result, to reach the highest resolution it is necessary either to use the optics with the small residual divergence, or to place the sample as close as possible to the detector.

We reached 15 microns (comparable with the CCD resolution) as the minimum allowed resolution since the used semilens had a residual divergence of $1.5 \mathrm{mrad}$ and the sample was placed about $15 \mu \mathrm{m}$ from the detector because of the physical dimensions of the stage rotation (Hampai et al. 2009, 2011).

We used as experimental parameters a CCD exposure time of $250 \mathrm{~ms}$, in order to avoid CCD saturation, while the sample was positioned on the xyz $\vartheta$ micro-positioner and step rotated by $0.5^{\circ}$ per image, for a total of 720 projection images, with a rotation velocity was $0.5 \mathrm{~s}^{-1}$ for a total exposition time of $720 \mathrm{~s}$.

In addition, to reduce the noise effects a background subtraction has been performed by the image acquisition software (Image-Pro Express). The reconstruction work is separated into two stages: the conversion of the projection images into the slice images (Octopus code) and the rendering process (Amira code). In Fig. 6, a 3D reconstruction with a corner cube cut is shown. The onion-geometry of all biological layers is clearly seen (Hampai et al. 2013).

\subsection{Dynamic computed tomography (DynamicCT)}

First studies concerning dense sprays investigation by means of X-ray absorption techniques date back to the 80s. In 1984 Gomi and Hasegawa (1984) estimated the mass distribution of the liquid phase in a water/gaseous nitrogen spray by the $\mathrm{X}$-ray absorption method. Recently, synchrotron radiation source (SRS) absorption-based techniques have been applied to investigate high-dense regions of the fuel sprays providing

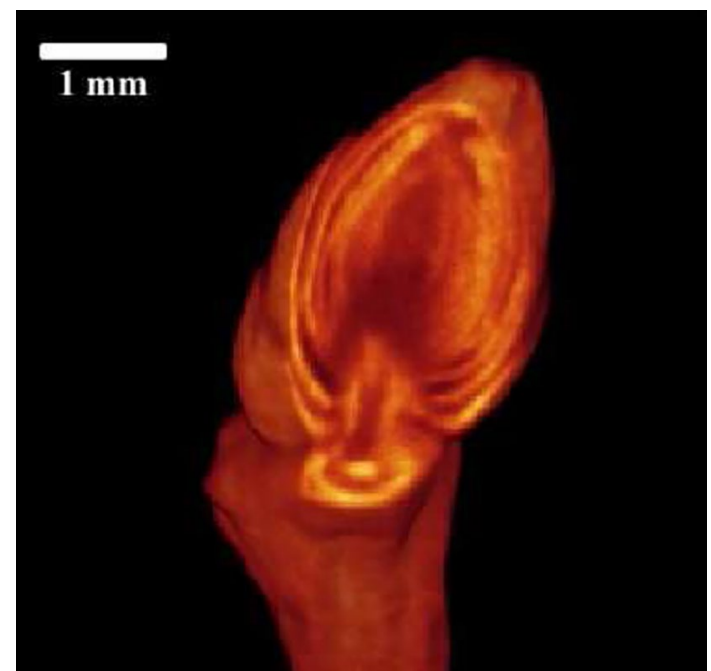

Fig. 6 X-ray micro Tomography of a flower bud. The experimental setup is based on polycapillary semilens with $1.4 \mathrm{mrad}$ residual divergence and the power source of $22 \mathrm{~W}$. To obtain this 3D image, 720 images on 360 with an exposition time of $250 \mathrm{~ms} / \mathrm{image}$ for a total exposition time of $12 \mathrm{~min}$ were registered and analysed (Gomi and Hasegawa, 1984)

quantitative measurements of the fuel mass (Im et al. 2013; Kastengren et al. 2008). X-rays penetrate the dense part of fuel spray because of their weak interaction with the low $\mathrm{Z}$ hydrocarbon chain. X-ray radiography and tomography have been used to investigate the core of gasoline and diesel sprays and to reconstruct the three-dimensional (3D) structure. SRS are high-brilliant sources providing monochromatic and collimated radiation beams with pulsed structure at high frequency.

Conventional X-ray sources are discarded with respect to SRS because of their lower energy, high divergence and time-continue structure (Kak and Slaney 1999). Recent progresses in advanced optics for X-ray applications such as polycapillary lenses have allowed overcoming some of these limits and then permitting to obtain high-intense quasi-parallel beams with a 60\% transmissivity (Marchitto et al. 2015).

The low divergent beam emerging from polycapillary optics enabled recording the images at extremely low intensities (without the necessity to increase the radiation intensity in order to get high $\mathrm{S} / \mathrm{N}$ ratio). Such set-up has permitted to study the peculiarities of the spray morphology by an easy-to-access and low-cost set up in comparison with synchrotron radiation-based apparatuses. Thanks to the axial and $3 \mathrm{D}$ rendering reconstructions of spray structures, the absorption profiles in the region near the nozzle hole have been drawn. The experimental calibration of gasoline X-ray absorption enabled the evaluation of the density profile along specified jets.

Figure 7 reports both fuel density and X-ray absorption distributions in the jet longitudinal plane. The experimental 

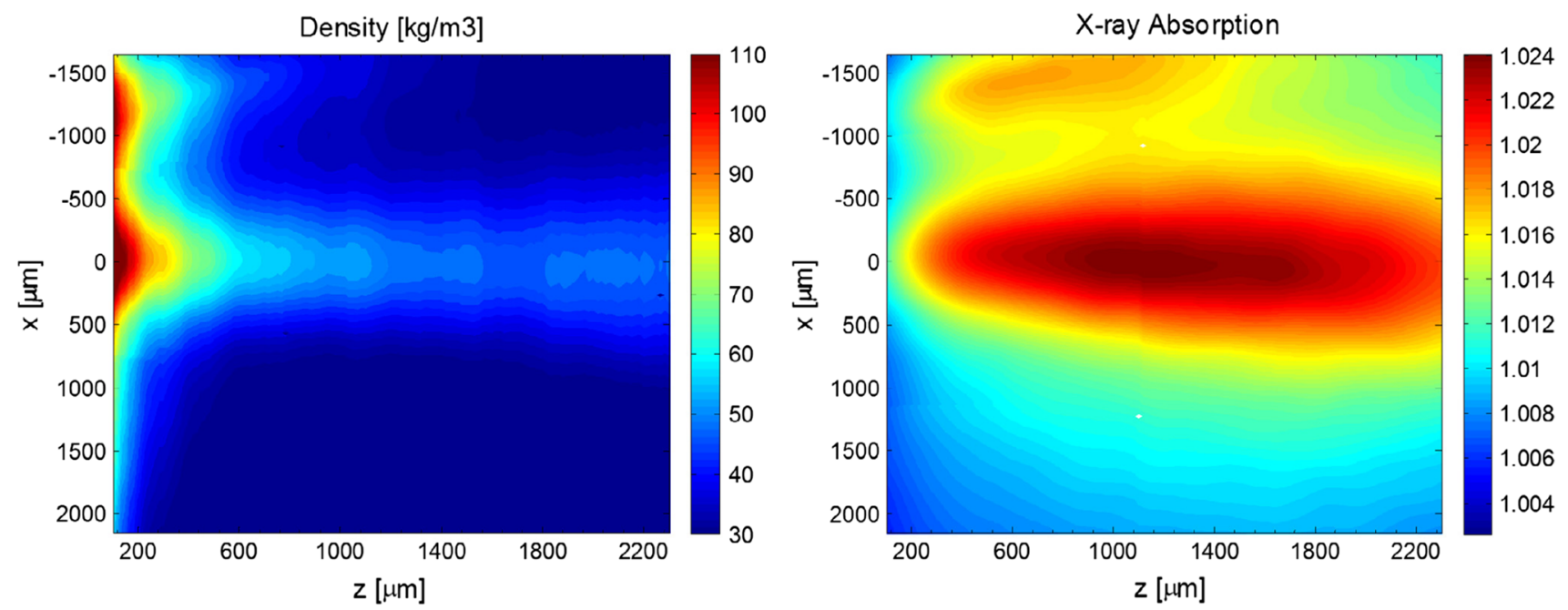

Fig. 7 Jet longitudinal plane distribution of fuel density (left) and X-ray absorption (right) (SPIE 2000). Experimental conditions: acquisition of 100 images for a time of $2 \mathrm{msec} / \mathrm{step}$ during a $360^{\circ}$ scan with a step of $0.1^{\circ}$

set-up was made of an Oxford Apogee Cu Kalpha X-ray tube working at $30 \mathrm{kV}$ and $1 \mathrm{~mA}$, with a focal spot size of 50 around 50 microns coupled with a polycapillary semi-lens (transmissivity of $60 \%$ for $\mathrm{Cu} \mathrm{X}$-rays and a residual divergence of about $1.4 \mathrm{mrad}$ ) to obtain a quasi-parallel beam. We acquired a set of 100 images for a time of $2 \mathrm{msec} / \mathrm{step}$ during a $360^{\circ}$ scan with a step of $0.1^{\circ}$.

$\mathrm{X}$ axis refers to the jet cross-section, while $\mathrm{y}$ axis is normal directed to the nozzle. The radiation absorption is a function of the crossed fuel mass, and its estimation results useful to better understand the jet profile as well as the corresponding density distribution.

At the nozzle hole exit, the absorption immediately increases due to the combined effect of the increase of both cross-section radius and density, up to the maximum at a distance of about $1200 \mu \mathrm{m}$ from the orifice. The related density profile shows a maximum just out of the nozzle. At $150 \mu \mathrm{m}$ from the nozzle the maximum value of $110.5 \mathrm{~kg} / \mathrm{m}^{3}$ has been detected. The fuel has been delivered at high pressure, and its density has been elevated. In this region the air-fuel interaction is significant only on the spray edge. Later, the signal decreases due to the combined effect of growing spray section, loss of momentum flux and enhanced air-fuel mixing.

\section{LabOS}

Since from its foundation to nowadays, the XlabF laboratory has acquired several instrumental devices that need to be managed through a dedicated framework system enabling also the remote access, when necessary. As a result, we designed and developed Vitruvio, based on a LabVIEW platform that allows a complete control of one or more experimental devices in parallel.

The central system core is the "operative units", composed of generic applications dedicated to the different tasks assigned to them through JSON format. The units are divided into four groups: Admin, COM, analysis and FE (front end) as shown in Fig. 8.

First, the Admin class units receive commands from users, that are subsequently processed and finally sorted with the purpose of allocating each action to the adequate level of allocation. Since the communication protocols between the various units are based on the JSON language, the user interfaces do not necessarily have to be on the LabVIEW platform, but can be completely generic.

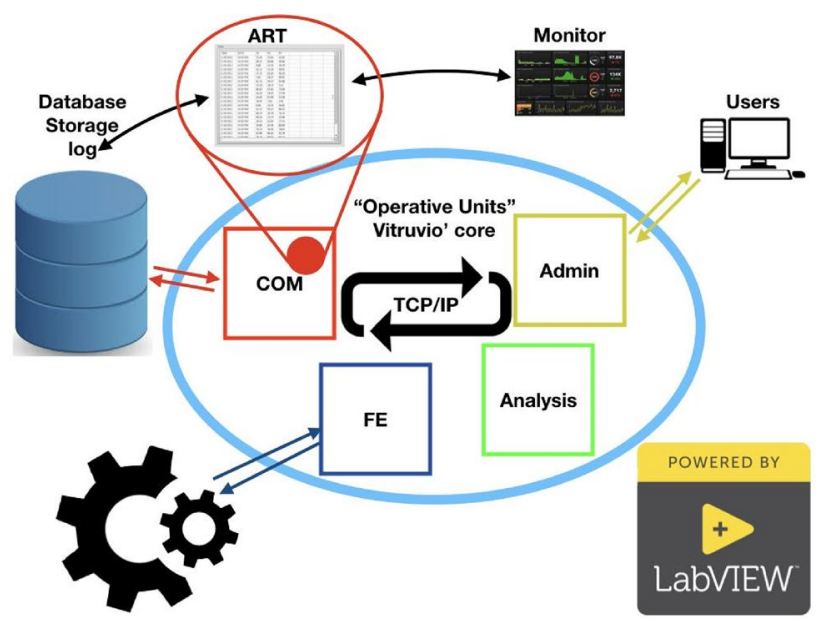

Fig. 8 A schematic of the Vitruvio layout, comprehending the four units and their interaction with external elements 
Table 2 Kinds of polycapillary optics produced @ XlabF and their characteristics

\begin{tabular}{|c|c|c|c|c|c|}
\hline Optics typology & Focal data & Size of single channel & Energy range & Transmission & PolyCO length \\
\hline $\begin{array}{l}\text { Full PolyCO } \\
\text { Lens } \\
\text { Semilens } \\
\text { PolyCO } \\
\text { Straight PolyCO } \\
\text { Full-semi MonoCO } \\
\quad \text { lens } \\
\text { Single capillary } \\
\quad \text { (shaped-full, semi- } \\
\text { straight) }\end{array}$ & $\begin{array}{l}30-80 \mathrm{~mm} \text { (focal } \\
\text { distance) } \\
60-90 \mu \mathrm{m} \text { (focal spot } \\
\text { of full lens) }\end{array}$ & $\begin{array}{l}\text { 3-10 } \mu \mathrm{m} \text { (PolyCO 4th } \\
\text { generation) } \\
>20 \mu \mathrm{m}(\text { MonoCO })\end{array}$ & $\begin{array}{l}\text { 3-40 keV (PolyCO4th } \\
\text { generation) } \\
1-5 \mathrm{keV} \text { (MonoCO) }\end{array}$ & $\begin{array}{l}40-70 \% @ 8 \mathrm{keV} \\
\text { (PolyCO 4th genera- } \\
\text { tion) } \\
20-40 \% @ 17 \mathrm{keV} \\
\text { (PolyCO 4th genera- } \\
\text { tion) }\end{array}$ & $\begin{array}{l}30-50 \mathrm{~mm} \text { Semilens } \\
60-100 \mathrm{~mm} \text { full lens } \\
\text { Inlet/outlet: } 3-10 \mathrm{~mm}\end{array}$ \\
\hline
\end{tabular}

The Analysis units contain all the analysis algorithms, that in our case where Vitruvo is applied to XlabF, are all algorithms for spectroscopic, diagnostic and characterization studies.

The FE class units are the interface with the hardware present in the laboratory. Thanks to the modularity of the units, we have the possibility to insert any device at any time, as in the case of a detector, a source or a remote movement control. Moreover, thanks to the JSON format, the hardware itself does not necessarily need or request a driver specifically written in LabVIEW language.

Finally, COM class units are used for managing and interfacing with storage and database systems. One unit in particular is dedicated to a separate operation: the ART unit is a matrix of information of the current state of the operating system, i.e. all the other units, and this matrix allows us to process a log (event log) and to view it on a control monitor. This is particularly useful in case of critical events to be evidenced and solved.

Therefore, Vitruvio, although it was conceived for the management of all the running systems of XlabF, is a definitely generic management system that is adaptable to all the needs of an automatized framework in a lab where instrumentations and machines work independently and in parallel.

\section{Technological transfer activities @ XChannel-technological polo}

The XlabF laboratory currently is the unique Italian laboratory dedicated to the design, manufacture and characterization of X-ray and neutron polycapillary optics (Table 2). Invented in 1984, the polycapillary lenses (the polycapillary optical systems are often called "polycapillary lenses") are composed of millions of hollow channels in a glass, in which the entering radiation is efficiently transmitted by multiple reflections, thanks to the phenomenon of total external reflection of the X-rays. This way, it is possible to collect the divergent radiation, focusing it or converting it into a parallel beam, and then model the beam as needed.

Given this unprecedented behaviour, the use of polycapillary optics open up the possibility of building brilliant X-ray sources with performances comparable to those normally reached with synchrotron radiation sources by combining a conventional X-ray tube with polycapillary lenses. X-ray capillary optics evolved from an attractive and simple idea into an independent area in optics that is being actively developed (SPIE 2000).

First polycapillary lens, about $1 \mathrm{~m}$ length and $30 \mathrm{~cm}$ diameter at larger transverse cross-section, was artificially manufactured in 1986 and allowed soft X-ray focusing at more than one-meter distance from X-ray source with one order increase of the radiation intensity at the lens focus. Since that a technology for fabricating monocapillaries and polycapillaries (and also composite and solid-drawn capillary systems) was created and is currently being perfected (so far there have been five generations of capillary lenses). In contrast to the ordinary methods of focusing X-ray radiation, polycapillary optics makes it possible to really handle various large divergent beams of X-rays produced by conventional X-ray tubes.

Presently, XlabF represents one of the few facilities all over the world able to realize polycapillary optics, thanks to the presence within the INFN National Laboratories of Frascati of the Polycapillary Technopolo (also named as XChannel-Technological Polo), dedicated to the whole production chain, ranging from fabrication of the lens/semilens from raw materials to the characterization of the final product. This was made possible thanks to the agreement signed by Prof. SB Dabagov with the Unisantis FZE for the acquisition of all the intellectual rights covering the production of the PolyCOs, and including both the know-how and the industrial apparatuses.

In addition, given the expertise and established know how of the XlabF researchers about polycapillary production, we are able to design and produce customised optics according to the users' experimental requirements. In the 
following tables, we summarise the characteristics of the different PolyCO currently available for experimental studies.

\section{Conclusions}

The XLab Frascati laboratory offers the possibility of conducting experiments with analytical techniques based on the application of X-rays, such as diffractometry and fluorescence, to study materials from both the elemental and structural point of view. In addition, the presence of a facility dedicated to X-ray imaging and tomography, coupled with the opportunity to use polycapillary optics (lens and semilens systems) enable users to perform 2D/3D reconstruction of a sample through the complementary analysis of their composition and even in the case of materials with a very low concentration of their components. The versatility of these powerful techniques allows their application throughout several different interdisciplinary sectors such as cultural heritage, biology, geology and industry with performances overcoming the limits presented by some techniques currently adopted in research laboratories (Dabagov and Gladkikh 2019; Dabagov 2018).

This is possible thanks to the use of the polycapillary optics that enables to create a very bright $\mathrm{X}$-ray source from a conventional one and then obtain a powerful quasi-parallel or a focused beam from a divergent source, according to a characteristic "semi-barrel" or "barrel" shape, respectively, and to the purpose of the analysis to perform. These are hollow optics composed of millions of developed glass channels that work as X-ray manipulator (as the radiation is transmitted through multiple reflection from the walls in every single channel of these optical curves), similar to what can be done with optical fibers with visible light, and they are manufactured at XChannel-Technological Polo, i.e. the technological facility present at XlabF and within the INFN-Laboratori Nazionali di Frascati currently producing customised lens/ semilens devices with peculiar capabilities that provide great opportunities with conventional table top X-ray layout.

Finally, we have designed the layout of the new X-ray tomography facility that will be equipped with a conventional X-ray microfocus source and polycapillary optics that is intended to target fields such as biology, geology and cultural heritage to offer the opportunity of making $\mathrm{X}$-ray studies with a very high resolution. It is planned to be built up in the next few months to be then operative and open to external users.

Acknowledgements One of the authors (SBD) would like to acknowledge the support by the Competitiveness Program of the National Research Nuclear University MEPhI (Moscow).

\section{Compliance with ethical standards}

Conflict of interest The authors declare that no conflict of interests exists.

\section{References}

Almaviva S, Bonfigli F, Franzini I, Lai A, Montereali RM, Pelliccia D, Cedola A, Lagomarsino S (2006) Hard X-ray contact microscopy with $250 \mathrm{~nm}$ spatial resolution using a LiF film detector and a tabletop microsource. Appl Phys Lett 89:054102

Baldacchini G, Bonfigli F, Faenov A, Flora F, Montereali RM, Pace A, Pikuz T, Reale L (2003) Lithium fluoride as a novel X-ray image detector for biological world capture. J Nanosci Nanotechnol 3:483

Bernini R, Pelosi C, Carastro I, Venanzi R, Di Filippo A, Piovesan G, Ronchi B, Danieli P (2016) Dendrochemical investigation on hexachlorocyclohexane isomers $(\mathrm{HCHs})$ in poplars by an integrated study of micro-Fourier transform infrared spectroscopy and gas chromatography. Trees Struct Funct 30:1455-1463

Bonfigli F, Faenov A, Flora F, Francucci M, Gaudio P, Lai A, Martellucci S, Montereali RM, Pikuz T, Reale L, Richetta M, Vincenti MA, Baldacchini G (2007) High-resolution water window X-ray imaging of in vivo cells and their products using LiF crystal detectors. Microsc Res Tech 71:35

Bostick BC, Theissen KM, Dunbar RB, Vairavamurthy MA (2005) Record of redox status in laminated sediments from Lake Titicaca: a sulfur K-edge X-ray absorption near edge structure (XANES) study. Chem Geol 219(1-4):163-174

Cappuccio G, Cibin G, Dabagov SB, Di Filippo A, Piovesan G, Hampai D, Maggi V, Marcelli A (2018) Challenging X-ray fluorescence applications for environmental studies at XLab Frascati. Condens Matter 3(4):33

Chang W, Kerner J, Franc E (2001) Analytical micro X-ray fluorescence spectrometer. Adv X-Ray Analytics 44:325-328

Community Members EPICA (2004) Eight glacial cycles from an Antarctic ice core. Nature 429:623-628

Cotton RA, Fletcher JH, Webb CE, Stead AD, Ford TW (1994) Comparison of laser-generated plasma X-ray sources for contact microscopy. In: Richardson MC (ed) Applications of laser plasma radiation, Proceedings of SPIE 2015, pp 86-96

Dabagov SB (2003) Channeling of neutral particles in micro- and nanocapillaries (reviews of topical problems). Phys Uspekhi 46(10):1053-1075

Dabagov SB (2018) Advanced Channeling technologies in plasma and laser fields (invited review). Eur Phys J Web Conf 167:01002

Dabagov SB, Gladkikh YuP (2019) Advanced channeling technologies for X-ray applications (invited review). Radiat Phys Chem 154:3-16 (online 15 June 2018)

Di Filippo A, Biondi F, Cufar K, de Luis M, Grabner M, Maugeri M, Presutti-Saba E, Schirone B, Piovesan G (2007) Bioclimatology of beech (Fagus sylvatica L.) in the Eastern Alps: spatial and altitudinal climatic signals identified through a tree-ring network. J. Biogeogr. 34:1873-1892

Fukuda Y, Faenov AY, Pikuz T, Kando M, Kotaki H, Daito I, Ma J, Chen LM, Homma T, Kawase K, Kameshima T, Kawachi T, Daido H, Kimura T, Tajima T, Kato Y, Bulanov SV (2008) Soft $\mathrm{X}$-ray source for nanostructure imaging using femtosecond-laserirradiated clusters. Appl Phys Lett 92:121110

Gogolev AS, Cherepennikov YuM, Vukolov AV, Rezaev RO, Stuchebrov SG, Hampai D, Dabagov SB, Liedl A, Polese C (2015a) WD-XRA technique in multiphase flow measuring. Nucl Instr Meth B 355:276-280 
Gogolev AS, Hampai D, Khusainov AKh, Zhukov MP, Dabagov SB, Potylitsyn AP, Liedl A, Polese C (2015b) Results of testing the energy dispersive Si detector with large working area. Nucl Instr Meth B 355:268-271

Gomi H, Hasegawa KI (1984) Measurement of the liquid phase mass in gas-liquid sprays by X-ray attenuation. Int J Multiph Flow 10:553-661

Hampai D, Cappuccio G, Cibin G, Dabagov SB, Sessa V (2007) Modeling of X-ray transport through polycapillary optics. Nucl Instr Meth A 580:85-89

Hampai D, Cherepennikov YuM, Liedl A, Cappuccio G, Capitolo E, Iannarelli M, Azzutti C, Gladkikh YuP, Marcelli A, Dabagov SB (2018) Polycapillary based $\mu$ XRF station for 3D colour tomography. J Instrum 13:C04024

Hampai D, Dabagov SB, Cappuccio G, Cibin G, Sessa V (2009) X-ray micro-imaging by capillary optics. Spectrochim Acta B 64:1180-1184

Hampai D, Dabagov SB, Cappuccio G, Longoni A, Frizzi T, Cibin G, Guglielmotti V, Sala M (2008) Elemental mapping and microimaging by X-ray capillary optics. Opt Lett 33(23):2743-2745

Hampai D, Dabagov SB, Della Ventura G, Bellatreccia F, Magi M, Bonfigli F, Montereali RM (2011) High resolution X-ray imaging by polycapillary optics and lithium fluoride detectors combination. EuroPhys Lett 96:60010

Hampai D, Dabagov SB, Polese C, Liedl A, Cappuccio G (2014) Laboratory total reflection $\mathrm{X}$-ray fluorescence analysis for low concentration samples. Spectrochim Acta B 101:114-117

Hampai D, Liedl A, Cappuccio G, Capitolo E, Iannarelli M, Massussi M, Tucci S, Sardella R, Sciancalepore A, Polese C, Dabagov SB (2017) 2D-3D XRF elemental mapping of archeological samples. Nucl Instr Meth B 402:274-277

Hampai D, Marchitto L, Dabagov SB, Allocca L, Alfuso S, Innocenti L (2013) Desktop X-ray tomography for low contrast samples. Nucl Instr Meth B 309:264-267

Im K-S, Cheong S-K, Powell CF, Lai M-CD, Wang J (2013) Unraveling the geometry dependence of in-nozzle cavitation in high-pressure injectors. Sci Rep 3(1):2067

Kak A, Slaney M (1999) Principles of Computerized Tomography Imaging. IEEE Press, NY

Kastengren AL, Powell CF, Riedel T, Cheong S-K, Im K-S, Liu X, Wang YJ, Wang J (2008) Nozzle geometry and injection duration effects on diesel sprays measured by X-ray radiography. J Fluids Eng. 130:041301

Kirz J, Jacobsen Ch (1995) Soft X-ray microscopes and their biological applications. Q Rev Biophys 28:33

Liedl A, Dabagov SB, Hampai D, Polese C, Tsuji K (2015) On X-Ray channeling in a vibrating capillary. Nucl Instr Meth B 355:289-292
Maggi V (1997) Mineralogy of atmospheric microparticles deposited along the Greenland Ice Core Project core. J Geophys Res 102:26725-26734

Marchitto L, Hampai D, Dabagov SB, Allocca L, Polese C, Liedl A (2015) GDI spray structure analysis by polycapillary X-ray $\mu$-tomography. Intern J Multiph Flow 70:15-21

Montereali R, Almaviva S, Bonfigli F, Cricenti A, Faenov A, Flora F, Gaudio P, Lai A, Martellucci S, Nichelatti E, Pikuz T, Reale L, Richetta M, Vincenti M (2010) Lithium fluoride thin-film detectors for soft X-ray imaging at high spatial resolution. Nucl Instr Meth A 623:758-762

Montereali RM (2002) Handbook of thin film materials (chapter 7). In: Nalwa HS (ed) Ferroelectric and dielectric thin films, vol 3. Academic, New York, pp 399-431

Moy ChM, Dunbar RB, Guilderson ThP, Waldmann N, Mucciarone DA, Recasens C, Ariztegui D, Austin JA Jr, Anselmetti FS (2011) A geochemical and sedimentary record of high southern latitude Holocene climate evolution from Lago Fagnano, Tierra del Fuego. Earth Planet Sci Lett 302:1-13

NANORAY (2011) NANORAY: a portable X-ray machine, FP7 Project N 222426 (2008-2011).

Petit JR, Jouzel J, Raynaud D, Barkov NI, Barnola J-M, Basile I, Bender M, Chappellaz J, Davisk M, Delaygue G, Delmotte M, Kotlyakov VM, Legrand M, Lipenkov VY, Lorius C, Pepin L, Ritz C, Saltzmank E, Stievenard M (1999) Climate and atmospheric history of the past 420,000 years from the Vostok ice core Antarctica. Nature 399:429-436

Polyak VJ, Asmerom Y (2001) Late holocene climate and cultural changes in the southwestern United States. Science 294:148-151

Ridolfi F, Renzulli A, Cerredo ME, Oberti R, Boiocchi M, Bellatreccia F, Della Ventura G, Menichetti M, Tassone A (2010) Amphibole megacrysts of the Cerro Jeu-Jepén pluton: new constraints on magma source and evolution (Fuegian Andes, Argentina), GeoSur2010. Applicata 51:80-83

SPIE (2000) Selected research papers on Kumakhov optics and applications. In: Proceedings of SPIE 4155 (2000)

Sawidis T, Breuste J, Mitrovic M, Pavlovic P, Tsigaridas K (2011) Trees as bioindicator of heavy metal pollution in three European cities. Environ Pollut 159:3560-3570

Publisher's Note Springer Nature remains neutral with regard to jurisdictional claims in published maps and institutional affiliations. 